\title{
Design of Remote Fetal Heart Rate Monitoring System based on Virtual Instruments
}

\author{
Zuliang Huang \\ Postgraduate \\ Shanghai University of \\ Engineering Science, \\ Shanghai 201620, China
}

\author{
Chen Deng \\ Professor \\ The correspondence author \\ Shanghai University of \\ Engineering Science, \\ Shanghai 201620, China
}

\author{
Dongdong Fei \\ Postgraduate \\ Shanghai University of \\ Engineering Science, Shanghai \\ 201620, China
}

\begin{abstract}
In view of the development of modern medical technology, we use LabVIEW software as a platform to design a kind of remote heart monitoring system. This monitoring system has realized the fetal heart rate data collection, transmission and storage. Using the network technology to connect the hospital center server, the system can check detection records, analyze and print the diagnosis in real-time, reach the aim of remote monitoring. Most functions of the system will be realized by software instead of traditional detection hardware, which will not only greatly reduce the cost of development but also improve the development efficiency.
\end{abstract}

\section{General Terms}

Virtual instrument; Remote monitoring; PC; Monitoring system.

\section{Keywords}

Virtual instrument; Fetal heart rate detection; Remote monitoring; Software design; Database.

\section{INTRODUCTION}

Virtual instrument is a kind of concept instrument, which replaces the traditional single measuring instrument by using the computer open architecture, it can process, display and store all kinds of data for computer. The prominent advantages of virtual instrument, on the one hand, it can combine with computer technology and use computer powerful functions as to process, display and store data, on the other hand, it can easily use network technology to share data. Virtual instrument can be installed in any of the computer and its function is stored in a computer's hard drive in the form of files. The software development platform is the key step of the design, LabVIEW is a widely used platform today.

For traditional fetal heart rate monitoring instrument, the signal gathering, signal processing and output displaying are implemented by the hardware circuit, but the circuits in the production requires high technology, the most part of them are introduced from abroad and expensive, it is also easy to cause the waste of resources to maintenance and update the circuits.
Based on the thought of "software is instrument"[1-2] in the virtual instrument technology of computer, through the software simulate the control panel and display of the traditional instrument, it can further realize multi-functional requirements, such as the signal analysis, a large amount of storage and network communication and so on. In recent years, virtual instrument system based on the LabVIEW development platform has been widely applied to various fields of biomedical engineering, this paper mainly introduces software design of the fetal heart rate monitoring system based on virtual instrument. The system is practical, a pregnant woman can complete remote fetal heart rate monitoring process without leaving home by installing terminal detection software on PC equipped with fewer hardware facilities, it also realize data sharing with the hospital central server by the network technology. The system is convenient for pregnant women daily fetal heart rate inspection, it timely and accurately reflect the dynamic of fetal and improves anomaly detection rate of fetal heart rate monitoring.

\section{THE OVERALL STRUCTURE OF THE SYSTEM}

Fetal heart rate monitoring system [3-6] is mainly composed of signal acquisition terminal, detection terminal, information processing system and care center. Figure 1 is the whole system structure. Signal acquisition terminal is the hardware device, it collects fetal heart rate of pregnant women and connects with the detection terminal; the detection terminal is a computer installed detection terminal software, it is responsible for the fetal heart rate signal acquisition, sending detection information, receiving diagnosis results, processing fetal heart rate data in a simple way and displaying them; The detection information and fetal heart rate data will be sent to the information processing system by sharing network, those will be analyzed and managed, and diagnosis results will be returned to the detection terminal at the end of the test; Care center receives detection information and fetal heart rate data from the sharing network, those are displayed in the monitoring interface and observed by a nurse, the heart rate data from the sharing network, those are displayed in the 
Pregnant women will send data to the hospital center server and receive diagnosis results.
Information processing system backups, analyses and manages the detection information and fetal heart rate data.

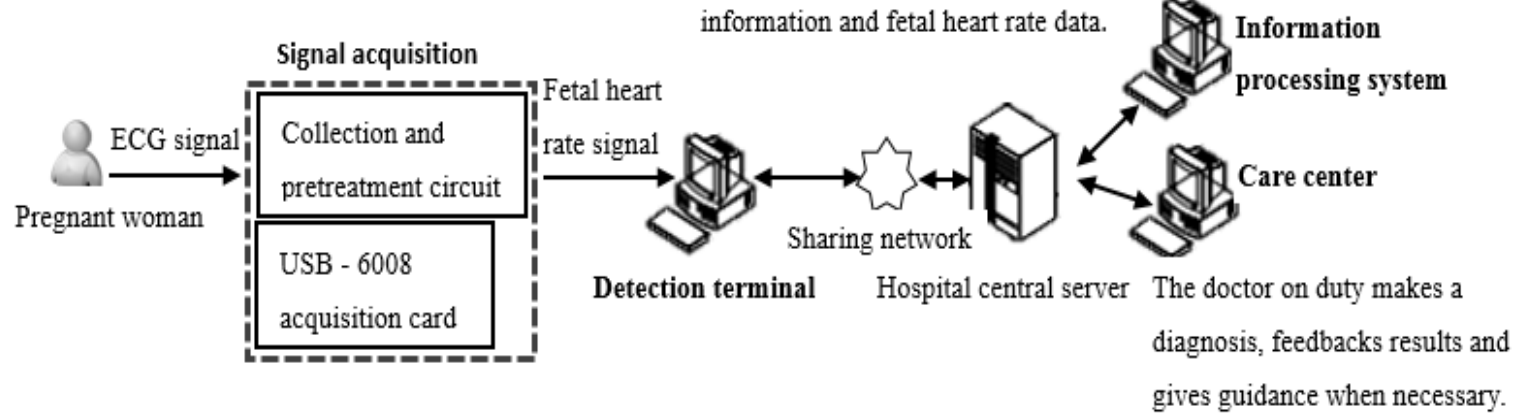

Fig 1: An overlook of the system

monitoring interface and observed by a nurse, the system will prompt the nurse when the data is abnormal, at this time, the medical staff can directly provide advice and guidance to pregnant women.

\section{THE HARDWARE DESIGN OF THE MONITORING SYSTEM}

By using the virtual instrument to strengthen the functions of software, the cost of system development can be greatly reduced. So the hardware of this system is simplified, only by two aspects: the pretreatment circuit and the USB - 6008 data acquisition card.

Collection and pretreatment circuit is the only needed hardware, the module adopts the medical ultrasound doppler technology to collect cardiac signal, the ultrasonic probe is the front detector, when it closes to the mother, the launch chip completes the work of transmit ultrasound, the receive chip receives the echo signal. Finally, the collection and pretreatment circuit outputs the processed fetal heart rate signal through detection, frequency selective amplification and bandpass filter processing etc.

USB-6008 acquisition card is a multi-function data acquisition card based on USB serial port, it has eight analog inputs that can be used for cardiac signal input, and each road has an input range of $\pm 10 \mathrm{v}$, a resolution of 12 bit. In addition, it also has multiple input and output of digital signal, which is used to control switch and the input. This module can use the software and hardware timing to capture, when using the software timing to capture, the module can adopt in 200 the dot/s sampling rate, it will convert the amplified analog signal into digital signal and send it to PC through USB interface for further processing.

\section{THE SOFTWARE DESIGN OF MONITORING SYSTEM}

The system uses LabVIEW 2011, which is a graphic language developed by the National Instrument Corporation. Microcomputers encode applications that is able to realize the real-time signal collection, transmission, analysis and storage with LabVIEW 2011. The characteristics of the system is the powerful signal processing ability of computer, network information sharing ability, large capacity data storage, it achieve the function of fetal heart rate signal analysis of guardianship. System function is shown in figure 2.

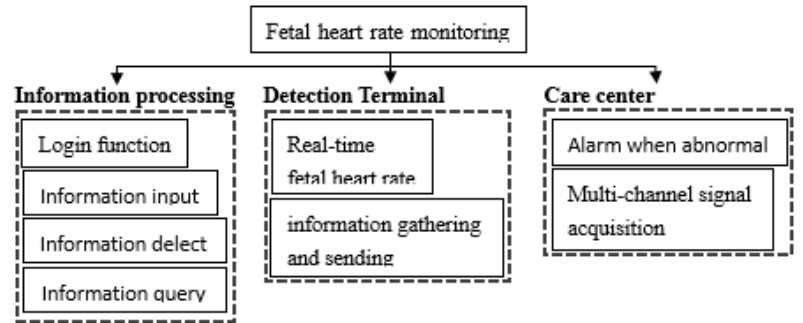

Fig 2: The software function modules

\subsection{Design of each Function Module}

(1) The administrator login: authorized management or medical staff use the software to manage database and file servers. First, the user input name and password, the front panel will display the user's login history after information verification. Users are divided into administrators and testers, the administrators have the privilege to add and remove users.

(2)The information input, delete, and query: it is necessary to input pregnant women's personal information that will facilitate the clear management and ordinary of hospital, and make fetal heart rate data consistent with some necessary static information; The query results include the basic personal information, history test information and fetal heart rate monitoring diagram;

(3) Information gathering and sending: to transmit fetal heart rate data of detection terminal and pregnant women information to the sharing network, inspection the end command to the host at the end of detection, the host will store detection information and fetal heart rate data in the database, or a special directory.

(4) Multi-channel signal acquisition: fetal heart rate information includes personal information, testing records and fetal heart rate data. Considering the large amount of data from monitoring system and high real-time requirements, the system uses the software algorithm to realize collection and storage of the multi-channel fetal heart rate signal, which improve the storage efficiency and real-time performance of the system.

\subsection{Software Design Thought}

The whole system is divided into three parts, the focus of each part is different, and each of them can run independently. The software design process is shown in figure 3 : 


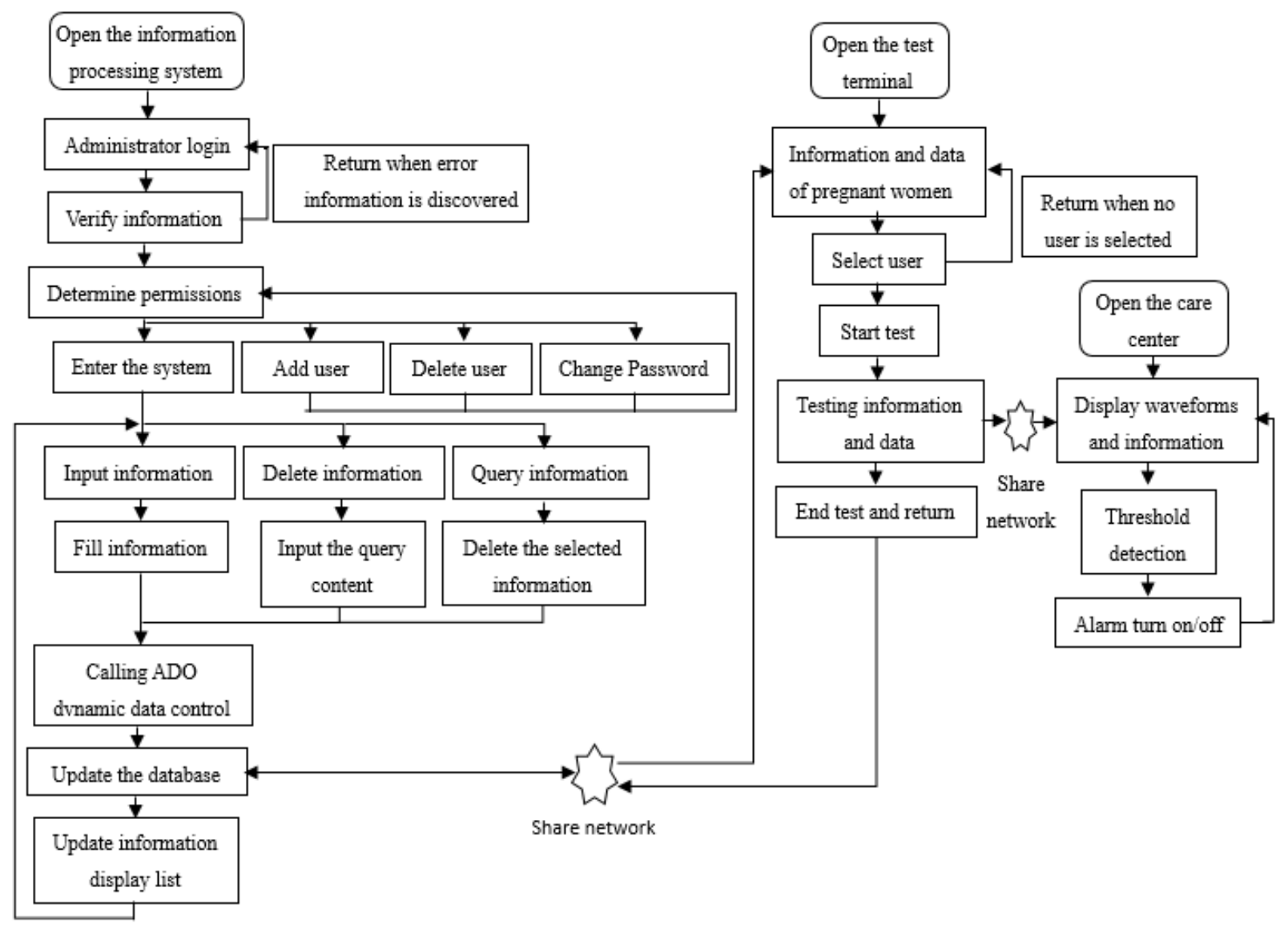

Fig3: design flow chart of heart monitoring system software

\section{THE KEY TECHNOLOGY}

\subsection{The Database}

LabVIEW development application software often need to access the database, especially to save the massive historical data in process control. Through the database access technology, users can easily create a database to manage a large amount of data, store process data and analyze the structure process of the system. But the LabVIEW does not provide directly connect to the database interface, does not have the function of database access. A variety of methods are mentioned in the literature [7] to accomplish the interface between LabVIEW and database, and compare the advantages and disadvantages of them. This system adopts the LabSQL to complete database access. LabSQL is a free, multiple databases, cross-platform LabVIEW database access kit, it supports the windows operating system that based on arbitrary ODBC database, including Access, SQLSever, Qracle, etc. This system takes advantage of LabSQL to access SQL and access database at the same time. Because of the amount of the administrator information data is small, and access can be run on the local computer, simple operation, so use access. The SQL Server is suitable for the processing of big data and has a good flexibility of background development as well as scalability. Therefore, it is also suitable for the storage of content in the form of multi tables.

\subsection{File server}

In order to accelerate the speed of data access, adopted the "index + folder" way of storage, The basic information of the pregnant women and file path index information stored in the database, and the fetal heart rate data stored in the specific directory structure of file server in the form of a file.
Receiving project of cardiac data store them after receiving complete data or file size is greater than the set value all at once, which reduces inventory in the process of receiving operation, speed up the transmission of data.

\subsection{Network communication}

It is mentioned in reference [8] that there are four kinds of methods that combine the LabVIEW with network technology. This system uses simple sharing variables to achieve network communication, through the network variables, users can easily realize the sharing of data between different computers with no programming required.

\subsection{The Monitoring Interface}

In order to solve the problem of small watch windows that is inconvenient for users when too many windows crowed in a limited space, we design the function of a magnifying glass that can be selectively amplified for a window alone. In order to observation of special requirements, we also design two monitoring methods of manual and automatic. Under the automatic monitoring, each of the on-line users set up independent watch window, real-time display the user's personal information, cardiac waveform, state monitoring etc. Under the manual monitoring, each online user enter the state, an independent monitoring window will be added when medical staff hits the button that make a terminal enter the monitored state.

\section{SOFTWARE IMPLEMENTATION}

The software design of fetal heart rate monitoring has been finished, we test the real-time data, the system administrator login, add/remove users, user information query, data manipulation, data monitoring and communication features. 
Function running stable and satisficing results are obtained. The interfaces of information processing system, detection terminal, care center are shown in figure 4, 5, 6 .

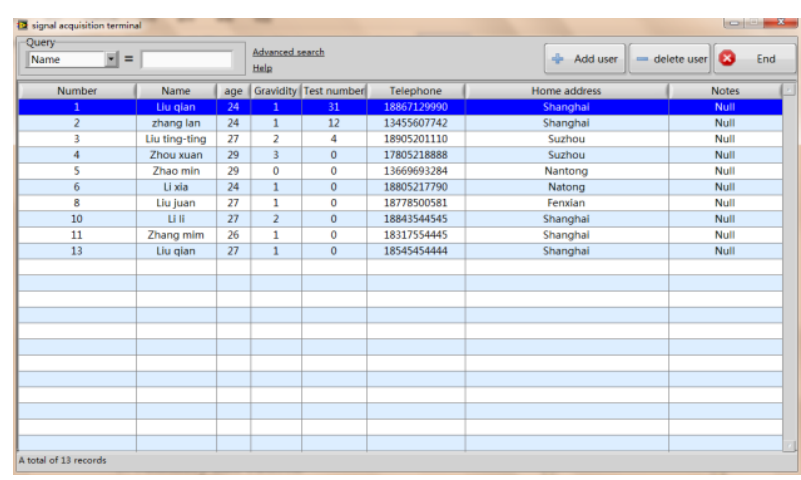

Fig 4: Information processing system interface

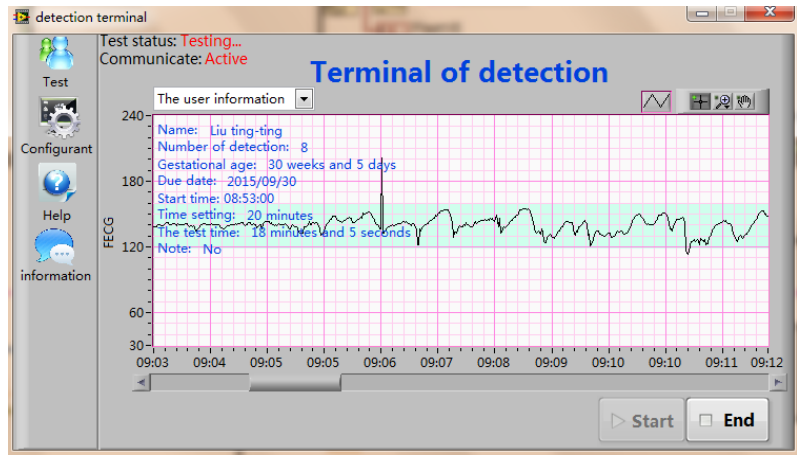

Fig 5: Detect the terminal interface

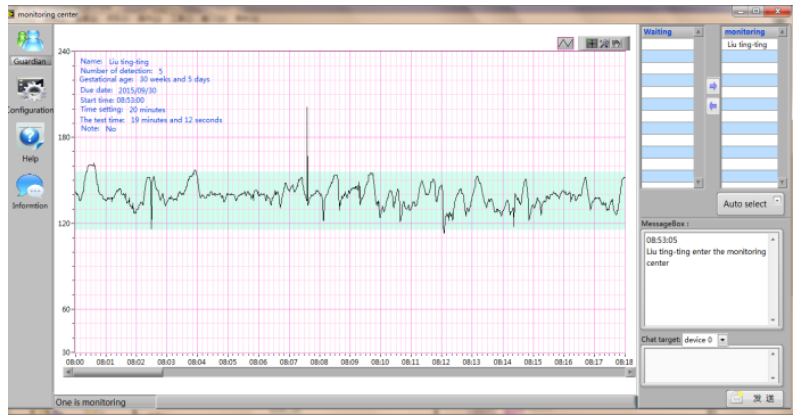

Fig 6: The center interface

\section{SUMMARY AND OUTLOOK}

The system uses LabVIEW 2011, which is a graphic language. The characteristic of the system is using the virtual instrument technology, which realized most of the fetal heart rate monitoring functions by the software instead of traditional hardware system, such as instrument panel control and display, a large amount of data storage, remote data transmission, etc. This design can lower the cost, reduce the difficulty of development, improve the efficiency of the guardianship, facilitate the expansion of the fetal heart rate monitoring in the future.

System has better modifiability and openness based on virtual technology, it can be easily modify functions or add a new function module. This system also needs to be improved in some aspects, such as the design of the handheld terminal [9], the network design of remote monitoring and off-line monitoring, etc. In recent years, with the rapid development of the virtual instrument technology, it has a very broad prospects for development in the field of medical instruments by its powerful features, a combination of both will go far.

\section{REFERENCES}

[1] Tong Zhang, Guoshun Chen, Zhenglin Wang. Proficient in LabVIEW programming $[\mathrm{M}]$. Electronic industry press, 2008.

[2] Xianglin Fang. LabVIEW and virtual medical instrument [J]. Journal of Biomedical Engineering, 2006, 25(1): 6566.

[3] Xiao Zhou, Ming Li, Jiajun Bu. The design and implementation of mobile remote medical monitoring system [J]. Computer engineering, 2010, 36 (10): 251253.

[4] Xinyou Ai, bin Xiao, Chengcheng Su. Remote heart monitoring system monitoring center software design [J]. China medical equipment information, 2011 (9): 18-21.

[5] Zhencheng Chen, Xiaoli Chen. The development of remote ecg monitoring system based on virtual instrument $[\mathrm{J}]$. Journal of medical and health unit, 2009, 30 (4): 19-21

[6] Kovacs Ferenc, Torok Miklos, Horvath Csaba. A new, phonocardiography - telemetric fetal based home monitoring system $[\mathrm{J}]$. Telemedicine journal and ehealth, 2014, 16(8):878-882

[7] Jihu Yin, Feng Wang. The LabVIEW database access technology based on LabSQL [J]. Instrument technology, 2011, (4): 55-56.

[8] Jian Liao, Xianjun Shi, Rong Zhang. Several kinds of method to realize network communication in LABVIEW [J]. Journal of instruments and meters, 2008, (8): 573576.

[9] Kai Yang, Hanjun Jiang, Zhihua Wang, etc. The Fetal heart rate monitoring system with mobile Internet [J]. Journal of Circuits and Systems (ISCAS), 2014:443-446 